\title{
Tabaquismo y Diabetes
}

\author{
NÉSTOR SOTO I.*
}

\section{Smoking and Diabetes}

Tobacco exerts damage in patients with diabetes mellitus by accelerating chronic complications, increasing the risk of global and cardiovascular mortality. Quitting smoking produces risk reduction. In addition, both active and passive smoking increases the incidence of type 2 diabetes. Exposure to tobacco during pregnancy can also predispose to the development of diabetes of daughters and gestational diabetes in mothers. A study in diabetes mellitus 1 compared a US cohort with a European cohort, showed that smokers have significantly higher levels of HbAlc and a lipid profile with a higher level of triglycerides and $L D L$ cholesterol. For all these reasons it is urgent to brief counsel or provide $A B C$-D, according to the First Clinical Practice Guidelines of Tobacco Treatment, Chile, 2017 for all diabetic patients, whether in outpatient or hospital care.

Key words: Smoking; tobacco smoke pollution; Diabetes Mellitus Type 2; Diabetes Gestational.

\section{Resumen}

El tabaco ejerce daño en los pacientes con diabetes mellitus acelerando las complicaciones crónicas, incremento en el riesgo de mortalidad global y cardiovascular. Dejar de fumar produce una reducción del riesgo. Además, el tabaquismo tanto activo como pasivo incrementa la incidencia de diabetes tipo 2. La exposición al tabaco durante el embarazo también puede predisponer al desarrollo de diabetes de las hijas y diabetes gestacional en las madres. Un estudio en diabetes mellitus 1 comparó una cohorte de EE. UU. con una europea, demostró que los fumadores tienen niveles de HbAlc significativamente mayores y un perfil lipidico con mayor nivel de triglicéridos y colesterol LDL. Por todas estas razones es urgente realizar consejería breve o ABC-D, según las Primeras Guías de Práctica Clínica de Tratamiento del Tabaquismo, Chile, 2017 a todo paciente diabético ya sea en atención ambulatoria como hospitalaria.

Palabras clave: Fumar; contaminación por humo de tabaco; Diabetes Mellitus tipo 2; Diabetes Gestacional.

El consumo de tabaco ejerce un efecto nocivo en los pacientes con diabetes mellitus (DM), acelerando las complicaciones crónicas, tanto micro como macrovasculares. El tabaquismo activo está asociado con un significativo incremento en el riesgo de mortalidad global y cardiovascular, así como el cese de fumar produce una reducción del riesgo. En un meta-análisis de 89 estudios de cohorte, el riesgo relativo ajustado asociado con el tabaquismo (intervalo de confianza de 95\%) fue $1,55(1,46-1,64)$ para mortalidad global, 1,49
(1,29-1,71) para mortalidad cardiovascular, 1,51 $(1,41-1,62)$ para enfermedad coronaria, 1,54 $(1,41-1,69)$ para accidente vascular encefálico y $2,15(1,62-2,85)$ para enfermedad arterial periférica ${ }^{1}$. Se estimó que un $14,6 \%$ del total de muertes en varones y $3,3 \%$ en mujeres fueron atribuibles a fumar entre los pacientes diabéticos. Aunque los exfumadores persistían con mayor riesgo de mortalidad total y cardiovascular comparados con los nunca fumadores, el aumento del riesgo fue significativamente más bajo, sugirien-

* Unidad de Endocrinología y Diabetes, Hospital San Borja Arriarán. Ex Presidente Sociedad Chilena de Endocrinología y Diabetes. 
do el beneficio de dejar de fumar en los pacientes diabéticos.

En el estudio finlandés de nefropatía diabética, que incluyó 3.613 pacientes con diabetes tipo 1 (DM1), se evaluó el riesgo acumulado a 12 años de microalbuminuria, macroalbuminuria y enfermedad renal terminal para fumadores actuales, ex fumadores y no fumadores. El riesgo de microalbuminuria fue $18,9 \%(\mathrm{p}<0,0001)$ para fumadores actuales y $15,1 \%(\mathrm{p}=0,087)$ para exfumadores comparados con $10,0 \%$ para no fumadores. Los riesgos para macroalbuminuria fueron $14,4 \%(\mathrm{p}<0,0001), 6,1 \%(\mathrm{p}=0,082)$ y $4,7 \%$, respectivamente y el de riesgo de enfermedad renal terminal fue $10,3 \%(\mathrm{p}<0,0001)$ para fumadores actuales y $10,0 \%(\mathrm{p}<0,0001)$ para exfumadores comparados con $5,6 \%$ para no fumadores. En los exfumadores, el riesgo de macroalbuminuria y enfermedad renal terminal no fue diferente al de los no fumadores después de ajuste multivariado, demostrando el beneficio de cesar el tabaquismo en esta población ${ }^{2}$.

Además del incremento en el riesgo de complicaciones, se ha observado que el tabaco incrementa la incidencia de diabetes tipo 2 (DM2), la cual también se encuentra elevada en fumadores pasivos. En un meta-análisis reciente ${ }^{3}$ que incluyó 88 estudios prospectivos con 5.898.795 participantes y 295.446 casos de diabetes tipo 2 incidente, el riesgo relativo combinado fue $1,37 \%$ (95\% IC 1,33-1,42) comparando fumadores actuales con no fumadores, $1,14(1,10-1,18)$ entre exfumadores con los nunca fumadores y 1,22 $(1,10-1,35)$ entre nunca fumadores con exposición versus sin exposición pasiva a humo. Además, se encontró una relación dosis respuesta. Comparados con los nunca fumadores, los riesgos relativos fueron 1,21 $(1,10-1,33)$ para fumadores livianos, $1,34(1,27-1,41)$ para fumadores moderados y $1,57(1,47-1,66)$ para fumadores pesados. Se estimó que $11,7 \%$ de los casos de DM2 en varones y $2,4 \%$ en mujeres fueron atribuibles a tabaquismo activo.

En otro meta-análisis destinado a estudiar especialmente a los fumadores pasivos, con 6 estudios prospectivos incluyendo 154.406 participantes, edades entre 18-74 años, con 7.116 nuevos casos de diabetes, los no fumadores que han estado expuestos al humo de tabaco en forma pasiva también presentaron un mayor riesgo que los no fumadores no expuestos al humo de tabaco (riesgo relativo combinado 1,21, 95\% IC 1,07-1,38) y tal asociación persistió en el análisis dosis-respuesta ${ }^{4}$.

En un estudio prospectivo de 1.801 hijas de 44-54 años, el tabaquismo materno prenatal tuvo una más fuerte asociación con el riesgo de diabetes de las hijas que el tabaquismo paterno, lo cual persistió después de ajustar por raza parental, diabetes y empleo. La estimación del efecto del tabaquismo parental no cambió cuando se ajustó por el peso de nacimiento de las hijas o el índice de masa corporal actual. El tabaquismo materno también se asoció significativamente con autoreporte de diabetes tipo 2 con un riesgo de 2,3 veces 5 .

En relación con el efecto sobre el control metabólico, un estudio en DM1 que comparó una cohorte de EE. UU. con una europea, demostró que los fumadores tienen niveles de $\mathrm{HbA} 1 \mathrm{c}$ significativamente mayores $(8,5 \%$ vs $7,9 \%)$ después de ajustar por edad, sexo, duración de DM1 y etnia. Los fumadores además tuvieron un perfil lipídico desfavorable con mayor nivel de triglicéridos y colesterol $\mathrm{LDL}^{6}$.

En el caso de DM2, el efecto del cese de tabaco es menos claro. Un estudio de cohorte retrospectiva de fumadores adultos con DM2 usando The Health Improvement Network (THIN), una gran base de datos de atención primaria en UK, investigó por modelos de regresión multiniveles ajustados la asociación entre la duración de la abstinencia de fumar, cambio en HbAlc y el efecto del cambio de peso corporal. De 10.692 adultos fumadores con DM2, 3.131 (29\%) cesaron y se mantuvieron en abstinencia al menos un año. Después de ajuste por potenciales confundidores, la HbA1c aumentó por $0,21 \%(95 \%$ IC $0 \cdot 17-0,25 ; \mathrm{p}<0,001)$; dentro del primer año después de suspender y disminuyó si la abstinencia continuaba, siendo comparable a la de los fumadores activos después de 3 años. Este aumento en $\mathrm{HbAlc}$ no fue mediado por cambio en el peso corporal ${ }^{7}$.

El consumo de nicotina puede producir reducción del peso corporal por estimulación directa del receptor de melanocortina (MC4-R), que resulta en disminución de la ingesta alimentaria. Además, disminuye los niveles de leptina y estimula el sistema nervioso simpático y la lipólisis. La disminución en la tasa de tabaquismo es paralela al aumento en la tasa de obesidad. Por otro lado, la nicotina puede disminuir la sensibilidad a insulina actuando sobre el sustrato del receptor de insulina 1 (IRS-1) ${ }^{8}$. Se ha visto que la insulinosensibilidad mejora con el cese de tabaco, lo que ocurre junto con normalización de la fosforilación del IRS-1; sin embargo, durante la cesación de tabaco se produce una disminución en el gasto metabólico sin un incremento simultáneo en la actividad física, por lo que es muy probable un balance positivo de energía que lleva a ganancia de peso. 
En una revisión sistemática se evaluaron 35 estudios de cohorte con 63.403 individuos que habían cesado de fumar y 388.432 fumadores. Los que pararon de fumar tuvieron un incremento de peso de 4,1 $\mathrm{kg}(95 \%$ CI 2,69-5,51; $\mathrm{p}<0,001)$ comparados a aquellos que continuaron fuman$\mathrm{do}^{9}$. Esto demuestra que la ganancia de peso es un efecto adverso mayor de cesar de fumar; sin embargo, el beneficio global sobre la salud sobrepasa con creces al riesgo del incremento de peso, ya que los fumadores de peso normal tienen mayor riesgo de mortalidad de todas las enfermedades relacionadas al tabaquismo que los ex fumadores que tienen sobrepeso $u$ obesidad ${ }^{10}$.

Por todas estas razones es imperioso que se realice consejería breve (ABC-D) a todo paciente diabético ya sea en atención ambulatoria como hospitalaria .

\section{Bibliografía}

1.- PAN A, WANG Y, TALAEI M, HU FB. Relation of Smoking with Total Mortality and Cardiovascular Events Among Patients with Diabetes: A Meta-Analysis and Systematic Review. Circulation 2015; 132: 1795 804.

2.- FEODOROFF M, HARJUTSALO V, FORSBLOM C, THORN L, WADÉN J, TOLONEN N, et al. Smoking and progression of diabetic nephropathy in patients with type 1 diabetes. Acta Diabetol 2016; 53: 525-33.

3.- PAN A, WANG Y, TALAEI M, HU FB, WU T. Relation of active, passive, and quitting smoking with incident type 2 diabetes: a systematic review and me- ta-analysis. Lancet Diabetes Endocrinol. 2015; 3: 95867.

4.- SUN K, LIU D, WANG C, REN M, YANG C, YAN L. Passive smoke exposure and risk of diabetes: a meta-analysis of prospective studies. Endocrine 2014; 47 : 421-7.

5.- LA MERRILL MA, CIRILLO PM, KRIGBAUM NY, COHN BA. The impact of prenatal parental tobacco smoking on risk of diabetes mellitus in middle-aged women. Journal of Developmental Origins of Health and Disease 2015; 6: 242-9.

6.- HOFER SE, MILLER K, HERMANN JM, DESALVO DJ, RIEDL M, HIRSCH IB, et al. International Comparison of Smoking and Metabolic Control in Patients With Type 1 Diabetes. Diabetes Care 2016; 39 : e177-8.

7.- LYCETT D, NICHOLS L, RYAN R, FARLEY A, ROALFE A, MOHAMMED MA, et al. The association between smoking cessation and glycemic control in patients with type 2 diabetes: a THIN database cohort study. Lancet Diabetes Endocrinol 2015; 3: 423-30.

8.- HARRIS KK, ZOPEY M, FRIEDMAN TC. Metabolic effects of smoking cessation. Nature Reviews 2016; 12: 299-308.

9.- TIAN J, VENN A, OTAHAL P, GALL S. The association between quitting smoking and weight gain: a systemic review and meta-analysis of prospective cohort studies. Obes. Rev 2015; 16: 883-901.

10.- SIAHPUSH M, SINGH GK, TIBBITS M, PINARD CA, SHAIKH RA, YAROCH A. It is better to be a fat ex-smoker than a thin smoker: findings from the 1997-2004 National Health Interview Survey-National Death Index linkage study. Tob. Control 2014; 23 : 395-402.

Correspondencia a:

Dr. Néstor Soto I.

Email: nsotoisla@gmail.com 